\title{
Auswirkung lokaler Bauwerksschäden auf die modalen Parameter
}

\author{
Entwicklung eines „Demonstrators“ (excel - Datei)
}

Univ.-Prof. DI Dr. Rainer Flesch, AIT Austrian Institute of Technology $\mathrm{GmbH} /$ Center for Mobility Systems, Wien und TU-Graz

Thomas Kramer, TU-Graz

\section{Kurzfassung}

Weltweit gibt es bereits seit Jahrzehnten Bemühungen, baudynamische Methoden zur Erfassung des aktuellen Tragwerkszustandes - entweder durch kontinuierliches Monitoring oder Untersuchungen in bestimmten Zeitintervallen - einzusetzen. AIT beschäftigt sich bereits seit ca. 1980 mit den obigen Themen. Im Kapitel 4 dieser Arbeit wird ein kurzer Überblick über die wichtigsten Projekte sowie die zugehörige Literatur geboten. Zufolge des zunehmenden Trends zum Building Information Modelling (BIM) wird es künftig im Fall von wichtigen Bauwerken immer leichter werden, gleichzeitig auch ein Referenzmodell für die dynamische Bauwerksinspektion vorzusehen. Die gegenständliche Arbeit beschreibt die Entwicklung eines „Demonstrators“ in Form einer Excel - Datei, mit der man die Auswirkung von lokalen Steifigkeitsminderungen auf die Eigenfrequenzen und Eigenvektoren darstellen kann. Der Demonstrator dient sowohl zur Veranschaulichung dieser Zusammenhänge im Baudynamik Unterricht als auch der Entwicklung neuer robuster model - updating - Verfahren.

\begin{abstract}
In many countries - since several decades - structural dynamics methods are applied for structural health monitoring either by continuous monitoring or measurements in regular intervals. AIT is active in this field since 1980. The most important projects are shortly mentioned in chapter 4. Due to the increasing application of BIM it will be possible in future to establish dynamic reference models for important structures and lifelines. The paper describes the elaboration of an excel - demonstator in order to show the influences of local damages at different story levels on eigenfrequencies and modeshapes. The demonstrator will be used for structural dynamics teaching as well as for the development of new robust model updating strategies.
\end{abstract}

Vortrag FLESCH/ KRAMER

2. Wiener Dynamik Tage 2021 


\section{Einleitung}

Weltweit gibt es bereits seit Jahrzehnten Bemühungen, baudynamische Methoden zur Erfassung des aktuellen Tragwerkszustandes - entweder durch kontinuierliches Monitoring oder Untersuchungen zu bestimmten Zeitpunkten bzw. in bestimmten Zeitintervallen einzusetzen. Diese Vorgangsweisen sind derzeit allerdings noch keine Standardverfahren und kommen nur gelegentlich zur Anwendung.

Es stehen heute bereits effiziente Möglichkeiten zur Erstellung von wirklichkeitsnahen Bauwerksmodellen für bestehende Bauwerke zur Verfügung. Es ist ein erstes 3D - FE Modell erforderlich, welches auf Basis der verfügbaren Bauwerksdokumente erstellt wird. Weiters werden dynamische insitu - Messungen durchgeführt und die maßgeblichen Eigenschwingzustände identifiziert. Als dynamische Anregung dient häufig die im Untergrund stets vorhandene „ambiente“ Schwingungsanregung bzw. die Anregung durch Verkehr oder durch eine künstliche Anregungsquelle (Impuls, Schwingungsgenerator). Das erste FE Modell wird anschließend an die Messergebnisse anpasst („model-updating“).

Der Aufwand hierfür erscheint insbesondere für wichtige Bauwerke, in denen sich viele Menschen befinden können, sowie für wichtige Infrastrukturbauten, die auch während und nach Katastrophen (z.B. Erdbeben) funktionstüchtig bleiben müssen, als gerechtfertigt. Es bestehen insbesondere folgende Anwendungsbereiche:

- „Structural Health Monitoring“ von Bauwerken, insbesondere von Brücken zur frühzeitigen Erkennung von Bauwerksschäden (Themen: Tragsicherheit und Erhaltungsmanagement)

- „Structural Assessment“: Beurteilung der Tragsicherheit und Gebrauchstauglichkeit von bestehenden Bauwerken, z.B. als Basis für geplante Umbauarbeiten von älteren Bauwerken, bei denen auch gleichzeitig die Erdbebensicherheit an den aktuellen Stand der Erdbebennormen angepasst werden soll.

AIT beschäftigt sich bereits seit ca. 1980 mit den obigen Themen. Im Kapitel 4 dieser Arbeit wird ein kurzer Überblick über die wichtigsten Projekte sowie die zugehörige Literatur geboten. AIT hat hierdurch wesentlich zur Weiterentwicklung des „model - updatings“ beigetragen. Es wurden insbesondere die Diplomarbeit von S. DEIX [42] und die Dissertation von $M$. RALBOVSKY [36] erarbeitet.

Drohnen sind derzeit bereits in der Lage, im Vorbeiflug die ambienten Schwingungsantworten von Bauwerken zu messen. Aus den gemessenen Zeitverläufen lassen sich die maßgeblichen

\section{Vortrag FLESCH/ KRAMER}

2. Wiener Dynamik Tage 2021 
Eigenfrequenzen bestimmen. Derzeit wird ein Forschungsprojekt geplant, in dem auf obige Weise Einsatzkräfte bei der Bergung von Personen aus beschädigten Gebäuden unterstützt werden sollen. Es geht hierbei um eine rasche Festlegung der optimalen Bergungsstrategie sowie den Schutz der Bergungsteams vor potentiellen Teileinstürzen des Bauwerks. Es besteht somit die Aufgabe, im Einsatzfall rasch ein passendes Bauwerksmodell zu erstellen, welches eine Abschätzung der vorliegenden Einsturzszenarien ermöglicht. Insbesondere für wichtige Infrastruktur sollte man in Zukunft für den unbeschädigten Zustand ein Referenzmodell erstellen, welches dann im Katastrophenfall einem raschen und effizienten „model-updating“ zu unterziehen wäre. Zufolge des zunehmenden Trends zum Building Information Modelling (BIM) wird es künftig immer leichter werden, gleichzeitig auch ein Referenzmodell für die dynamische Bauwerksinspektion vorzusehen. Um dann für die beschädigte Struktur rasch ein Modell zur Abschätzung der Resttragfähigkeit erstellen zu können, ist die Weiterentwicklung von schnellen und robusten model - updating-Strategien nötig, die sehr wahrscheinlich auch die Verwendung einfacher Ersatzstabmodelle einschließen.

Besonders große und gute Chancen zur Quantifizierung und Lokalisierung von Bauwerksschäden besteht natürlich, wenn im Bauwerk z.B. in jedem Geschoß Sensoren eines Schwingungs-Monitoringsystems installiert werden, die $\mathrm{zu}$ jedem Zeitpunkt eine Systemidentifikation erlauben. Die erforderliche Schwingungsanregung wird durch ambiente Schwingungen, Wind und Verkehr geliefert. Will man auch die besonders aussagekräftigen höheren Eigenschwingzustände für die Identifikation heranziehen, könnte man sogar im obersten Stockwerk einen relativ kostengünstigen Schwingungsgenerator installieren, mit dem man zu jedem gewünschten Zeitpunkt eine Anregung mittels Frequenzsweep vornehmen könnte.

Die gegenständliche Arbeit beschreibt im Hauptkapitel 5 die Entwicklung eines „Demonstrators“ in Form einer Excel - Datei, mit der man die Auswirkung von lokalen Steifigkeitsminderungen auf die Eigenfrequenzen und Eigenvektoren darstellen kann. Der Demonstrator dient sowohl zur Veranschaulichung dieser Zusammenhänge im Baudynamik Unterricht als auch der Entwicklung neuer robuster model - updating - Verfahren.

\section{Vortrag FLESCH/ KRAMER}

2. Wiener Dynamik Tage 2021 


\section{Konzept für die Anwendung baudynamischer Methoden}

Bei dieser Vorgangsweise nutzt man die Tatsache, dass zufolge von Bauwerksschäden Änderungen der Eigenschwingzustände (Eigenfrequenzen + zugehörige Eigenvektoren auch Eigenformen genannt -, Bauwerksdämpfung) auftreten. Im optimalen Fall sind diese Parameter für das unbeschädigte Bauwerk (Referenzzustand) bekannt. Durch den Vergleich der Parameter für den unbeschädigten und den beschädigten Zustand kann man das Auftreten von Schäden erkennen. Weiters versucht man, die Schäden zu quantifizieren und möglichst auch zu lokalisieren.

Ein Schaden führt in der Regel zu einer lokalen Steifigkeitsminderung, die ein Absinken der betroffenen Eigenfrequenzen bewirkt. Betroffen sind jene Eigenschwingzustände, deren Eigenformen im Schadensbereich große Krümmungen aufweisen. Liegt ein Schaden in einem Schwingungsknoten einer bestimmten Eigenform, ändert sich die zugehörige Eigenfrequenz nicht. Hieraus folgt, dass zufolge der unterschiedlichen Eigenformen zumindest theoretisch die Möglichkeit für eine Schadens-Lokalisierung besteht. Die wesentlich komplexeren Änderungen der Eigenvektoren können z.B. mit dem geplanten Demonstrator untersucht und anschaulich dargestellt werden.

Rand- und Lagerungsbedingungen (Auflager, Bodenfedern etc.) haben meist große Auswirkungen auf bestimmte Eigenschwingzustände.

Schäden führen weiters zu einem Ansteigen der Dämpfung. Es sei darauf verwiesen, dass diese Tatsache noch kaum zur Interpretation von Änderungen genutzt wird. Hier gibt es folgende Ansatzpunkte für künftige Untersuchungen:

- Interpretation der "globalen“ Zunahme der Dämpfungszahlen

- Anregungsstrategien zum Auffinden eines höheren lokalen Dämpfungsanstiegs bei Annäherung an die Schadensstelle

- gezielte Interpretation der gemessenen komplexen Eigenformen (meist wird ja nur der Realteil betrachtet!), der Imaginärteil repräsentiert das aktuelle Dämpfungsverhalten.

Für die dynamische Bauwerksinspektion werden die maßgeblichen Eigenschwingzustände (Eigenfrequenzen + zugehörige Eigenformen) des gegenständlichen Bauwerks herangezogen. Es ist ein erstes 3D - FE Modell erforderlich, welches auf Basis der verfügbaren Bauwerksdokumente erstellt wird. Weiters werden dynamische insitu -

\section{Vortrag FLESCH/ KRAMER}

2. Wiener Dynamik Tage 2021 
Messungen durchgeführt und die maßgeblichen Eigenschwingzustände identifiziert. Gemessen werden die dynamischen Bauwerks-Antwortschwingungen (Zeitverläufe der Schwinggeschwindigkeit bzw. der Schwingbeschleunigung). Bei künstlicher Anregung (Schwingungsgenerator, Impuls) wird zusätzlich der Zeitverlauf der anregenden Kraft gemessen. Aus den Antwortschwingungen werden die Eigenfrequenzen, Eigenvektoren (Eigenformen, mode-shapes) und modalen Dämpfungszahlen identifiziert. Falls auch die gemessene Erregerkraft vorliegt, erhöht sich die Aussagekraft der Ergebnisse deutlich.

In den letzten Jahren werden abgesehen von den dynamischen BauwerksAntwortschwingungen und den wichtigen Tragwerks-Temperaturmessungen weitere Sensoren eingesetzt, um z.B. Verschiebungen, Neigungsänderungen und Dehnungen als weitere Parameter für die Modellverbesserung zu erhalten.

Das erste FE - Modell wird anschließend an die Messergebnisse anpasst („model-updating“). Diese Anpassung erfolgt über eine Minimierung der Differenzen zwischen den berechneten und gemessenen Eigenfrequenzen und Eigenvektoren. Sehr häufig werden zur Vereinfachung der Vorgangsweise nur die Differenzen der Eigenfrequenzen herangezogen. Selbstverständlich müssen aber die zugehörigen gemessenen und berechneten Eigenformen qualitativ beachtet werden um sicherzustellen, dass zusammengehörende Eigenfrequenzen miteinander verglichen werden.

Vor der Modellverbesserung müssen jene Eingabeparameter festgelegt werden, die mit den größten Unsicherheiten behaftet sind (z.B. Bodenfedern, Randbedingungen, etc.). Diese Parameter stellen dann die „update - Parameter“ dar, deren Gesamtzahl möglichst gering gehalten werden soll, z.B. $\leq 10$. Häufig werden unter anderem die Moduli der einzelnen Materialien als update - Parameter angesetzt.

Für jeden update - Parameter muss die Sensitivität der einzelnen Eigenschwingzustände auf Änderungen der jeweiligen update-Parameter ermittelt werden. Bei Berücksichtigung von etwa drei bis sechs Eigenschwingzuständen kann man bereits meist gute Ergebnisse erzielen. Da die Zusammenhänge nichtlinear sind, ist eine iterative Vorgangsweise zur Berechnung der update - Parameter erforderlich. In jedem Iterationsschritt wird ein lineares Gleichungssystem gelöst, welches die jeweiligen Zwischenstände der update-Parameter ergibt.

Häufig verzichtet man in der Praxis auf ein theoriebasiertes Anpassungsverfahren und beschränkt sich auf Ausprobieren (trial and error).

Höhere Eigenschwingzustände besitzen in der Regel eine zunehmende Zahl von Bereichen mit hoher modaler Krümmung. Hierdurch steigt die Wahrscheinlichkeit, dass ein lokaler

\section{Vortrag FLESCH/ KRAMER}

2. Wiener Dynamik Tage 2021 
Schaden in einem solchen Bereich liegt und sich hierdurch die Chancen zur Schadenslokalisierung erhöhen. Diese höheren Eigenschwingzustände werden jedoch meist nicht durch ambiente Erregung bzw. Verkehrsanregung aktiviert. Zur Anregung ist ein Schwingungsgenerator mit der Möglichkeit zur Durchführung von Frequenzsweeps - gezielt in Punkten, wo diese Eigenformen große Schwingungsamplituden besitzen (z.B. MoSeS/ AIT) - erforderlich.

Grundsätzlich ist anzumerken, dass auch der Einfluss der Temperatur auf die Eigenfrequenzen beachtet werden muss. Beim Langzeitmonitoring muss stets auch die Temperaturen in relevanten Tragwerkspunkten gemessen werden. Bei Messungen zu bestimmten Zeitpunkten müssen die aktuellen Tragwerkstemperaturen beachtet werden. Es dürfen nur Messungen miteinander verglichen werden, die bei annähernd gleichen Tragwerkstemperaturen erfolgten.

Weiters ist anzumerken, dass die dynamischen Messungen stets bei relativ geringen Schwingungsamplituden erfolgen. Die Materialien werden keinesfalls bis in den nicht-linearen Bereich hinein beansprucht. Bereits bestehende Schäden stellen sich bei den Messungen als eine abgeminderte äquivalente linearisierte Steifigkeit („verschmierte reduzierte Steifigkeit“) dar.

\section{Verfahren zum "model-updating"}

Das Prinzip des Model-Updatings wurde bereits im Kapitel 2 erläutert und wird noch detaillierter von Göge und Link in [31] dargestellt. Das Ziel des Verfahrens ist es, selektierte Eigenschaften des Berechnungsmodels so anzupassen, dass das berechnete Tragwerksverhalten möglichst dem gemessenen Tragwerksverhalten entspricht. Die folgende Zusammenstellung wurde von M. Ralbovsky erstellt und ist in [30] enthalten.

Das Model-Updating kann auf verschiedenen Daten beruhen, z.B. ambiente dynamische Messungen, Messungen mit erzwungenen Schwingungen, Messung von statischen Verformungen bei bekannten Lasten, usw. Zusätzlich gibt es bei jedem Typ der Messung verschiedene Methoden der Auswertung. Doebling, et.al. [32] hat einen Überblick der schwingungsbasierten Identifikationsmethoden erstellt, wovon das Model-Updating Verfahren ein Teil ist. Die vorhandenen Methoden konzentrieren sich großteils auf Ergebnisse ambienter Messungen. Diese Methoden wurden von Maeck in [33] behandelt.

\section{Vortrag FLESCH/ KRAMER}

2. Wiener Dynamik Tage 2021 
Um das Model-Updating umsetzen zu können, muss zuerst das Differenzmaß J zwischen Berechnungsmodell und gemessener Realität definiert werden. Eine verbreitete Formulierung ist in Gleichung (1) dargestellt:

$$
J=\sum\left(\mathbf{z}_{\mathbf{c}}-\mathbf{z}_{\mathbf{m}}\right)^{T} \mathbf{W}\left(\mathbf{z}_{\mathbf{c}}-\mathbf{z}_{\mathbf{m}}\right)
$$

Hier ist:

$\mathrm{J}$ Zielfunktion

$\mathrm{Z}_{\mathrm{c}} \quad$ Vektor der berechneten Eigenschaften

$Z_{m} \quad$ Vektor der gemessenen Eigenschaften

W Gewichtungsmatrix.

Im Vektor der Eigenschaften sind die Eigenfrequenzen und Eigenformen inkludiert. Die Gewichtungsmatrix stellt die Gewichtung einzelnen Parameter sicher. Es wird empfohlen, die Matrix W als Diagonalmatrix aufzubauen, mit quadrierten Kehrwerten der Standardabweichung einzelner Parameter in der Diagonale:

$$
W_{i i}=\frac{1}{\sigma_{i i}^{2}}
$$

Die Standardabweichung sollte die Genauigkeit der messtechnischen Ermittlung der jeweiligen Eigenschaft beschreiben. Somit wird sichergestellt, dass die einzelnen Parameter gemäß ihrer Genauigkeit gewichtet werden.

In der Literatur lassen sich auch viele andere Formulierungen der Zielfunktion finden, wie z.B. in der Publikation von Alvin [34], die auf Ermittlung von Kraftresiduen basiert. Hier werden aus Kombination der Modellmatrizen und der gemessenen Schwingeigenschaften die Kraftresiduen in den Resonanzen quantifiziert:

$$
\begin{aligned}
& R_{G, r}=K^{-1} \cdot\left[\left(K-\omega_{m, r}^{2} \cdot M\right) \cdot \phi_{m, r}\right] \\
& J=\sum_{r} R_{G, r}{ }^{T} \cdot R_{G, r}
\end{aligned}
$$

$\begin{array}{ll}R_{G, r} & \text { Vektor der generalisierten Kraftresiduen } \\ \omega_{m, r} & r \text {-ter gemessene Eigenwert } \\ \phi_{m, r} & r \text {-te gemessene Eigenform }\end{array}$

Vortrag FLESCH/ KRAMER

2. Wiener Dynamik Tage 2021 
Die eigentliche Modellanpassung ist ein mathematisches Optimierungsproblem, bei dem die Zielfunktion J durch Änderung der Modellparameter minimiert wird. Die Beschreibung der Optimierungsprozeduren erfolgt u.a. in den Publikationen von Göge [31] und Alvin [34] sowie von Ralbovsky [36].

Weitere Informationen zur indirekten Systemidentifikation finden sich z.B. im Abschnitt 16.3 in [1] bzw. in [40]. Die indirekte Identifikation dient der Korrektur von Rechenmodellen mit Hilfe der Ergebnisse von Versuchsmodellen. Die indirekte Identifikation beginnt mit einem Vergleich des Rechenmodells mit dem Versuchsmodell. Im Fall des dynamischen Verfahrens zur Bauwerksinspektion werden die indirekten Modellparameter (die modalen Parameter) verglichen. Ist die Struktur des Rechenmodells hinreichend genau, dann ergibt sich das korrigierte Rechenmodell durch Parameteranpassung.

Die Anpassung kann auf verschiedenen Genauigkeitsniveaus erfolgen [41]:

- durch globale Anpassung über den E-Modul. Sämtliche Unsicherheiten Im Modell (Massendichte, Querschnittsabweichungen, unklare Randbedingungen) werden hier stellvertretend durch den korrigierten E-Modul abgedeckt. Diese einfache Vorgangsweise hat sich in einigen Projekten überraschend gut bewährt.

- durch die „trial-and -error“ Methode, falls sich die größten Modellunsicherheiten in einem bestimmten, lagemäßig bekannten Bereich konzentrieren.

- Durch systematische Methoden (siehe z.B. [40]).

Falls das Rechenmodell eine größere Anzahl von Freiheitsgraden als das Versuchsmodell besitzt, muss für den direkten Vergleich ein bezüglich der Freiheitsgrade reduziertes Rechenmodell aufgestellt werden. Hierzu kann ein vereinfachtes Rechenmodell oder eine Kondensation (siehe [40]) vorgenommen werden.

Bevor die Parameteranpassung erfolgen kann, muss die Zuordnung zwischen den Eigenschwingungsgrößen des Versuchsmodells und des Rechenmodells gewährleistet sein. Hierfür sind die Eigenfrequenzen alleine nicht ausreichend, es müssen zusätzlich -zumindest qualitativ - die Eigenformen betrachtet werden. Falls weitere Unsicherheiten bestehen, sollten die zugehörigen generalisierten Massen miteinander verglichen werden. Falls der globale Vergleich nicht genügt, muss tiefer ins Detail gegangen werden (z.B. Vergleich der generalisierten Massen von Subsystemen).

\section{Vortrag FLESCH/ KRAMER}

2. Wiener Dynamik Tage 2021 
Das korrigierte Rechenmodell unterscheidet sich vom Rechenmodell durch die Wahl freier Parameter (Korrekturansatz).

Die Korrektur aller Elemente der Systemparameter-Matrizen unter Beachtung ihrer Symmetrie wäre sehr aufwändig. Es wären bei voll besetzten Matrizen $3\left(n^{2}+n\right) / 2$ Werte zu korrigieren. Es kann daher eine globale, faktorielle Korrektur der Systemparameter Matrizen von Teilen des Systems durchgeführt werden [40]. Für die faktorielle Korrektur werden die zu schätzenden Parameter verwendet. Meist beschränkt sich die Korrektur auf die Steifigkeitsmatrix. Der Korrekturansatz lautet in diesem Fall (analoges Aussehen ggf. für die Massen- bzw. Dämpfungsmatrix):

$$
\left[K^{K}\right]=\left[K^{\prime}\right]+\sum_{l=1}^{L} a_{K, l}\left[K_{l}\right]
$$

mit

$\left[\mathrm{K}^{\mathrm{K}}\right] \quad$ korrigierte Steifigkeitsmatrix

[K'] Teilmatrizen, die nicht korrigiert werden

$\left[K_{l}\right]$ Teilmatrizen, die korrigiert werden.

Falls nur die Steifigkeitsmatrix korrigiert wird gilt für den Korrekturvektor

$$
a^{\top}=a^{\top} K=\left(a_{k, 1} \ldots . . a_{k, L}\right) \text {. }
$$

Seine Bestimmung erfolgt z.B. nach der Methode der gewichteten kleinsten Fehlerquadrate [40]. Die Korrekturparameter werden meist auf iterative Weise bestimmt.

Vor der Durchführung der Korrektur muss aufgrund der Kenntnisse über die Ungenauigkeiten des Rechenmodells und der Ergebnisse einer Empfindlichkeitsanalyse festgestellt werden, welche Systemparameter - Matrizen korrigiert werden sollen. Der Erfolg der Korrektur hängt wesentlich von der Wahl der Submatrizen ab.

In [41] wird die indirekte Systemidentifikation aus der Sicht der Anwendung zur Brückeninspektion betrachtet. Insbesondere für die folgenden Teilbereiche bestehen Modellierungsunsicherheiten und somit die Voraussetzungen zum Ansatz von zu korrigierenden Teilmatrizen:

- Torsionssteifigkeiten

- Steifigkeiten in den Koppelungsbereichen zwischen Paralleltragwerken

- Steifigkeiten im Fundierungsbereich.

Vortrag FLESCH/ KRAMER

2. Wiener Dynamik Tage 2021 
Weiters sei auf den Abschnitt 16.5 in [1] Direkte Ermittlung von Steifigkeiten durch dynamische in-situ Versuche verwiesen. Hier werden zunächst die Grundlagen zur Ermittlung des komplexen Eigenwertproblems (mit Laplace Variabler und komplexen Eigenformen) dargestellt.

Weiters wird die Residuenmatrix [ $r_{\mathrm{K}}$ ] erläutert und ihr Zusammenhang mit den Eigenvektoren $\left\{u_{K}\right\}$ dargestellt:

$$
\left[r_{K}\right]=A_{K}\left\{u_{K}\right\}\left\{u_{K}\right\}^{T}
$$

mit

$\left\{u_{k}\right\}^{\top} \quad$ transponierter Eigenvektor $\left\{u_{k}\right\}$

$A_{k} \quad$ Skalierungsfaktor. Falls der Eigenvektor $\left\{u_{k}\right\}$ auf die generalisierte Masse

$M_{K}{ }^{*}=1$ normiert wird gilt $A_{K}=1 / \omega_{K}$

$\omega_{\mathrm{K}} \quad \mathrm{k}$-te Eigenkreisfrequenz.

Falls die Eigenformen mit guter Genauigkeit aus den Messergebnissen identifiziert werden konnten, kann die Nachgiebigkeitsmatrix [F] wie folgt errechnet werden:

$$
[F]=[K]^{-1}=\left[\sum_{k=1}^{m} \frac{r_{K}}{\omega_{K}}\right]=\left[\sum_{k=1}^{m} \frac{\left\{u_{K}\right\}\left\{u_{K}\right\}^{T}}{\omega_{K}^{2}}\right]
$$

\section{Assessmentprojekte von AIT und TU-Graz}

1) Health Monitoring von Brücken. Inst. Für Stahlbetonbau/ Dr. K. Kernbichler. Einsatz der Losenhausen - Erreger. Vornahme künstlicher Tragwerksschädigungen. Brücke Raach, Gänstorbrücke Ulm/ DYWIDAG, div. Weitere Brücken, tw. in Ungarn (1981 - 1990).

Weiters Brenner Autobahngesellschaft/ Ing. Wietek. 1988 - 1989 Untersuchung der Brücken TÜ Obernberg, Gschnitztalbrücke, 3. Nößlachbrücke, Luegbrücke/ Rahmen 5. Publikationen [4] bis [14].

2) 1997-1998: Brite Euram Projekt BRPR-CT96-0277 System Identification to Monitor Civil Engineering Structures (SIMCES): insitu Vers. TÜ-Warth, TÜ Grosram. Künstl. Schädigungen am Abbruchobjekt Z24/ Schweiz. Österr. Partner Inst. Betonbau/ TU Graz unter Prof. Lutz Sparowitz (Projektmitarbeiter Bernhard Freitag, Bernhard Stebernjak). Publikationen [16] bis [20].

Vortrag FLESCH/ KRAMER

2. Wiener Dynamik Tage 2021 
3) 1999 - 2001: Environment \& Climate Project ENV4-CT97-0574 (DG 12 - EHKN), Advanced methods for assessing the seismic vulnerability of existing motorway bridges (VAB), Project coordinator: P1: arsenal research, R. Flesch, Austria. Im Mittelpunkt Talübergang Warth/ A2. Pseudodynamische Versuche ELSA/ JRC Ispra. Publikationen [21] bis [23].

4) 2004-2007: IP LESSLOSS/ SP 5 In-situ Assessment, Monitoring and Typification of Buildings and Infrastructures. Erarbeitung des European Manual for in-situ Assessment of Important Existing Structures. Sub-Project Coordinator: P1: arsenal research, Austria, R. Flesch. Publikationen [3], [28], [29].

5) 2004 über Vermittlung durch Univ.-Prof. L. Sparowitz von KAGES beauftragt: Ermittlung der Erdbebensicherheit der Krankenhäuser Knittelfeld, Stolzalpe und Leoben unter Berücksichtigung des EC8. Die Untersuchungen erfolgten vor der Planung von Umbau- und Sanierungsarbeiten. Unvergesslich bleiben die „Modellierungskünste“ von Prof. V. Benko/ Bratislava, der für uns das Modell des Kinderhauses/ Spital Leoben mit hoher Präzision modelliert hat.

Es wurde weiters bewiesen, dass - ausgehend vom optimierten linearen Startmodell - eine ingenieurmäßige iterative Vorgangsweise zur Erfassung des globalen nicht-linearen Verhaltens des Bauwerks unter Erdbeben angewendet werden kann. Auf diese Weise lässt sich die „Kollaps-Belastung“ des Gebäudes ermitteln. arsenal research (bzw. AIT) arbeitete dann in weiterer Folge bei den im Zuge des Umbaus erforderlichen Erdbebennachweisen vorbildlich mit den planenden Ziviltechnikern zusammen (Dr. M. Ralbovsky). Publikationen [24] bis [26], [30].

6) 2010 Nachweis der Erdbebensicherheit einer Verbrennungsanlage für radioaktive Abfälle vor und nach einem Umbau. Publikationen [30], [39].

\section{Entwicklung eines Demonstrators - Anwendung auf Hochbaumodelle}

\subsection{Vorgangsweise}

Vortrag FLESCH/ KRAMER

2. Wiener Dynamik Tage 2021 
Zunächst wird ein 10 geschossiger ausgesteifter Stockwerksrahmen mit Kern betrachtet. Die Geschoßhöhe beträgt einheitlich 3,0 m. Der Grundriss des Regelgeschosses ist in Abbildung 5-1 dargestellt.

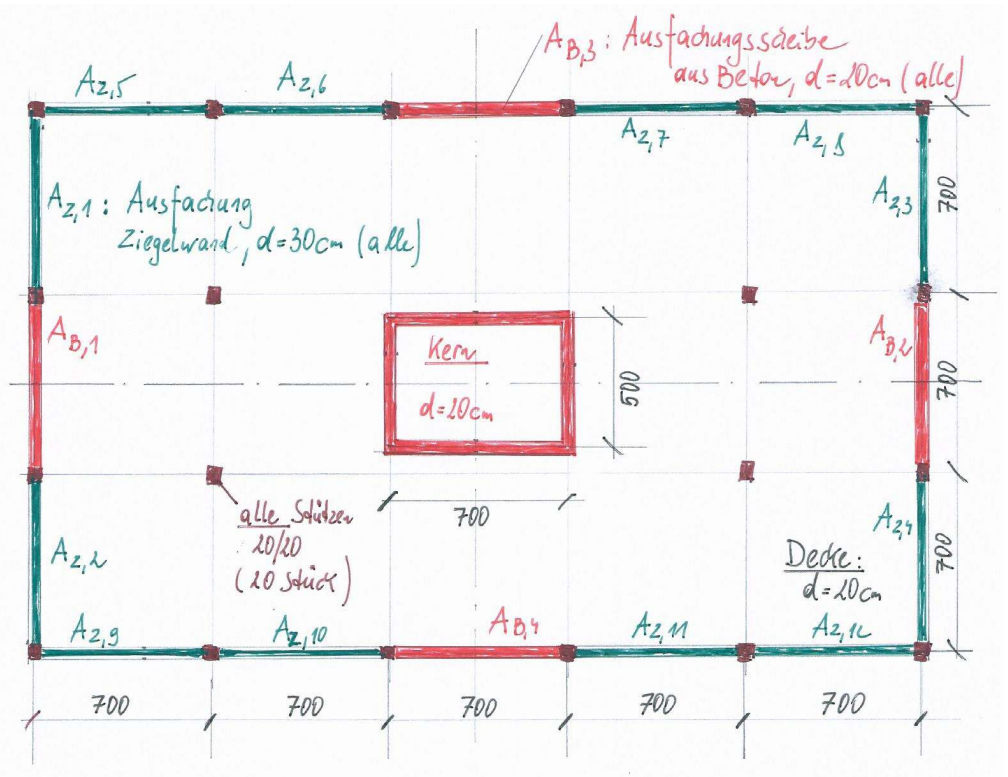

Abbildung 5-1: Grundriss Regelgeschoß

Es sind Aussteifungen durch Stahlbeton - Wandscheiben sowie Ziegel - Ausfachungswände vorhanden. In der vorliegenden Arbeit wird das Verhalten in Y- Richtung (schmälere Bauwerksseite) untersucht. Als Hauptfreiheitsgrade werden die horizontalen Verschiebungen in den 10 Stockwerkshorizonten dargestellt. Das Gebäude wird als Ersatzstab - Modell, welches die Biege- und die Schubverformungen berücksichtigt, abgebildet. Es werden in diese Richtung die wirksamen Biegesteifigkeiten und Schubflächen des Kerns, der 24 Stiele sowie der 2 Stahlbeton - Wandscheiben und der 4 Ziegel - Ausfachungswände berücksichtigt. Die Schubflächen und Trägheitsmomente der Ziegelwände werden hierbei unter Verwendung des Verhältnisses der Moduli auf eine äquivalente Stahlbeton - Wandscheibe umgerechnet.

Die Massenmatrix wird als Diagonalmatrix aufgestellt.

Die Berechnungen erfolgten zunächst mit dem FE-Programm SOFISTIK (siehe Abschnitt 5.2). Weiters wurde unter Anwendung des FE - Konzepts in einer Excel - Datei eine analytische Vorgangsweise umgesetzt. Ein erstes Beispiel wird im Abschnitt 5.3.1 präsentiert. Die weiteren Schritte zur Erarbeitung des Demonstrators, welche gerade durchgeführt werden, sind im Abschnitt 5.3.2 beschrieben.

\section{Vortrag FLESCH/ KRAMER}

2. Wiener Dynamik Tage 2021 


\subsection{Verwendung des FE - Programms SOFISTIK}

Das Bauwerk wurde als räumlicher Ersatzstab unter Berücksichtigung der Biege- und Schubverformungen modelliert. Es wurden alle Freiheitsgrade außer der Verschiebung in $\mathrm{Y}-$ Richtung und der Verdrehung um die $X$ - Achse gesperrt.

Zunächst wird das unbeschädigte Bauwerk mit gleichförmiger Steifigkeits- und Massenverteilung untersucht.

In der Folge wurden dann nacheinander Steifigkeitsminderungen in jedem der 10 Stockwerke simuliert, indem die jeweiligen Biegesteifigkeiten und Schubflächen (zunächst unter Annahme einer symmetrischen Beschädigung) um bestimmte Prozentsätze abgemindert wurden. Für jedes Stockwerk wurde hierbei eine um 30\% abgeminderte äquivalente Biegesteifigkeit und eine um $40 \%$ abgeminderte äquivalente Schubfläche für den Ersatzstab angesetzt (,verschmierte Vorgangsweise“). Massenänderungen zufolge der Schäden (z.B. Absturz von Füllmauerwerk) wurden zunächst nicht modelliert.

Bei der Bearbeitung mit SOFISTIK wurden für jede Variante 8 Eigenfrequenzen und Eigenformen ermittelt, um sie später denjenigen Ergebnissen gegenüberstellen zu können, die im Demonstrator aus der kondensierten Nachgiebigkeitsmatrix ermittelt werden.

In der Folge werden erste Ergebnisse präsentiert. In Abbildung 5.2 ist die absolute Abnahme der Eigenfrequenzen zufolge der stockwerksweise aufgebrachten Beschädigungen dargestellt. Aus der Legende ist die jeweilige Eigenfrequenz im unbeschädigten Zustand ersichtlich. Die horizontale Achse beschreibt das Stockwerk, in dem die Beschädigung angenommen wurde.

Vortrag FLESCH/ KRAMER

2. Wiener Dynamik Tage 2021 


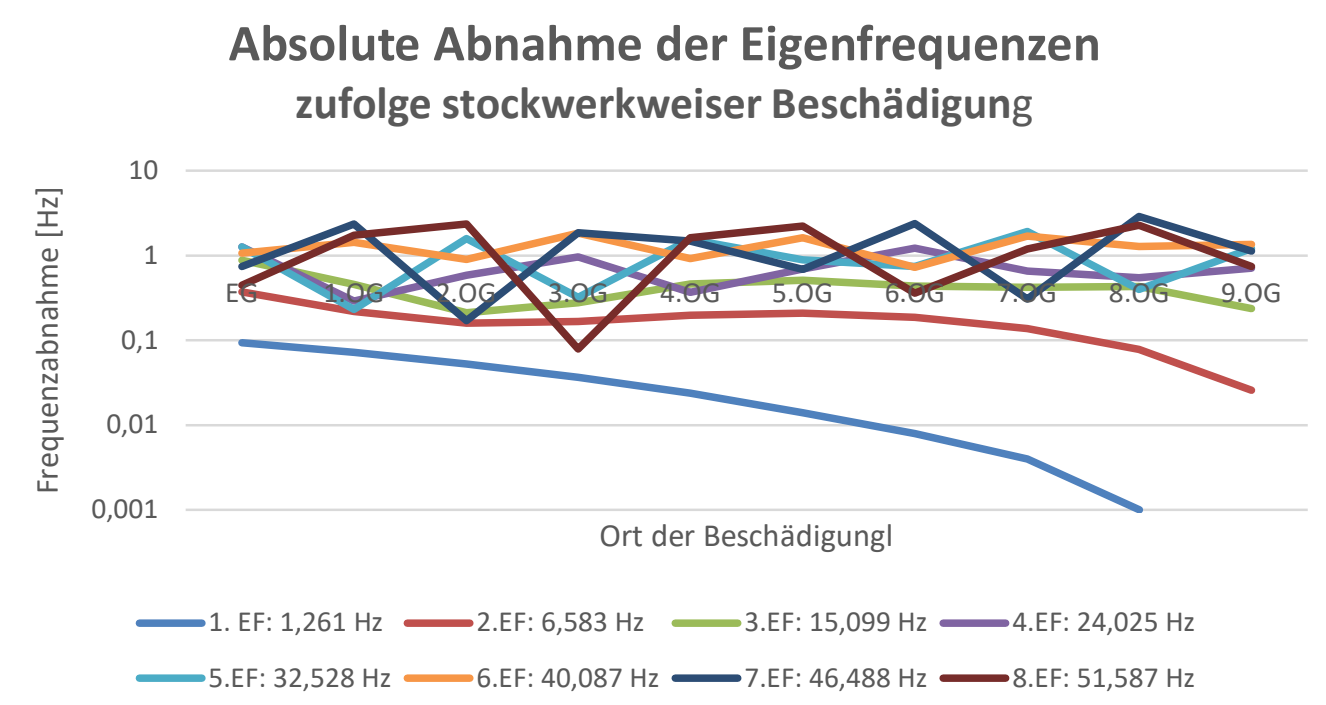

Abbildung 5-2: Absolute Abnahme der Eigenfrequenzen zufolge stockwerksweiser Beschädigung.

Zusätzlich sind in Abbildung 5.3 die relativen Abnahmen der Eigenfrequenzen dargestellt.

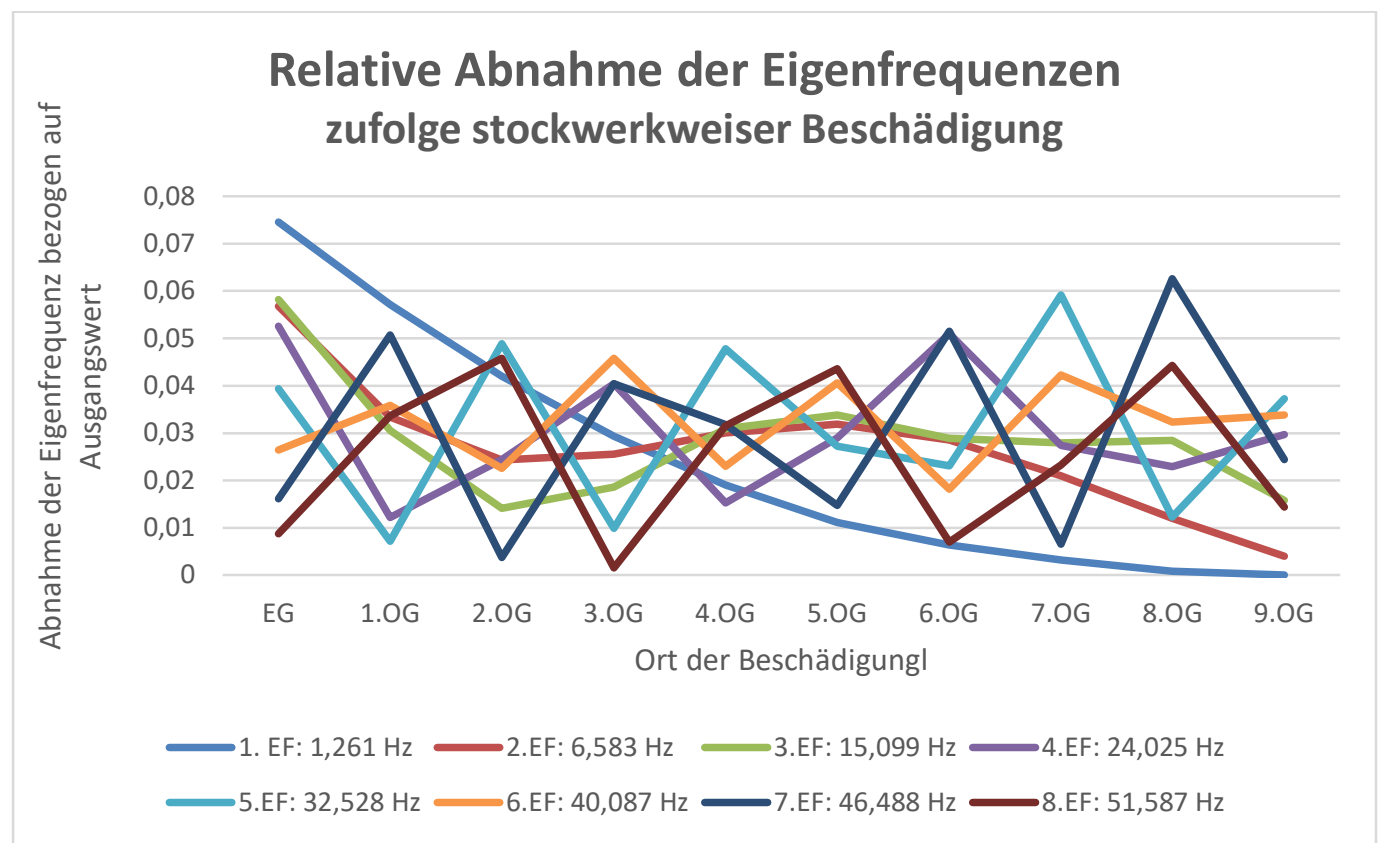

Abbildung 5-3: Relative Abnahme der Eigenfrequenzen zufolge stockwerksweiser Beschädigung.

Vortrag FLESCH/ KRAMER

2. Wiener Dynamik Tage 2021 
Weiters wurde zunächst der dritte Eigenschwingzustand ausgewählt, um die Auswirkungen der lokalen Schäden darzustellen. In Abbildung 5.4 wird die Änderung der 3. Eigenform zufolge der zehn Schadensszenarien dargestellt.

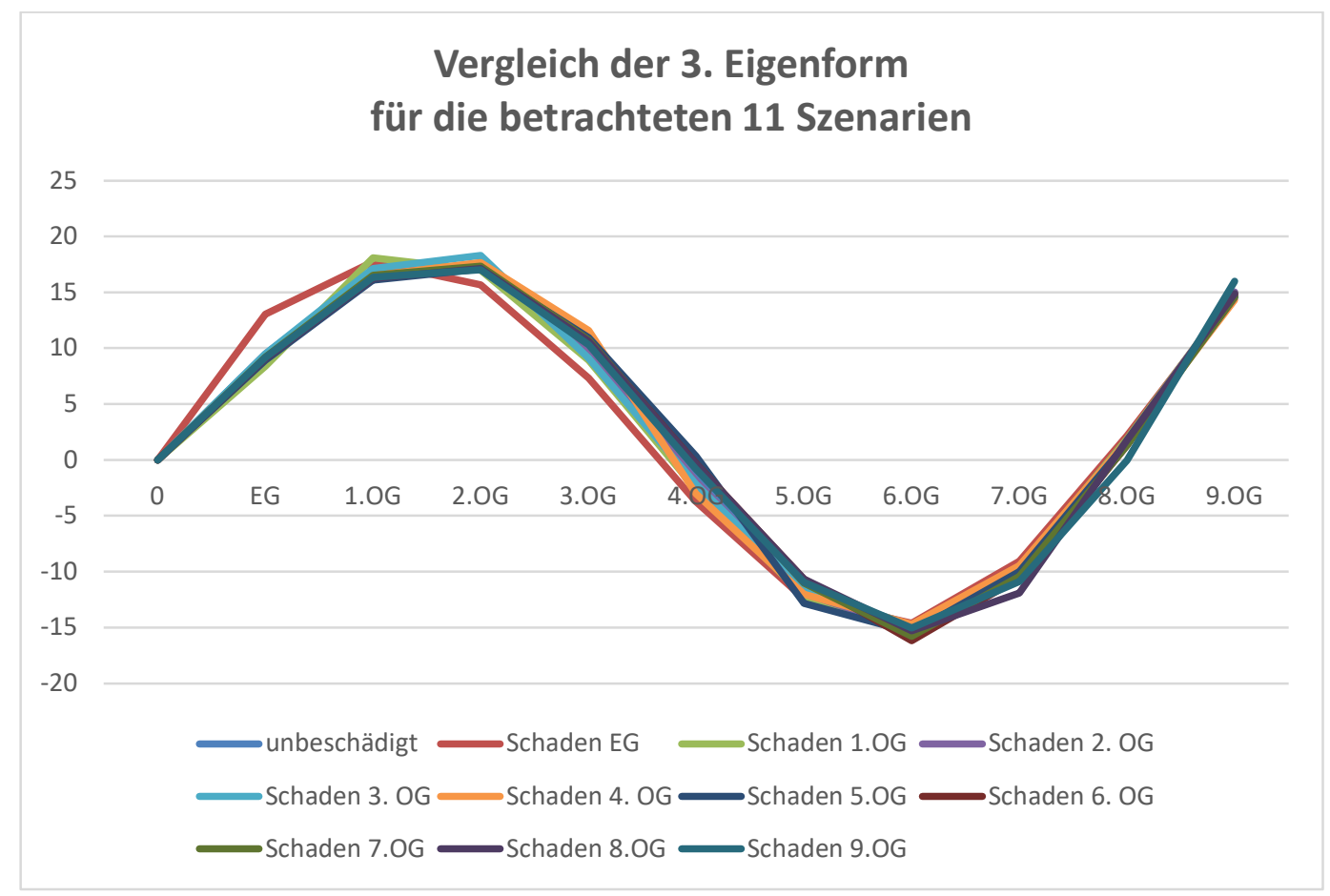

Abbildung 5-4: Vergleich der 3. Eigenform für die betrachteten 11 Szenarien

Weiters werden in Abbildung 5.5 die Änderungen der Amplituden in den einzelnen Stockwerken (beschrieben durch die horizontale Achse) dargestellt. Aus dieser Abbildung ist gut ersichtlich, in welchen Stockwerken deutliche Änderungen zufolge der einzelnen Schadensszenarien auftreten. Bei einem Bauwerk mit Monitoringsystem bestehen reelle Chancen, derartige Änderungen tatsächlich zu identifizieren. 


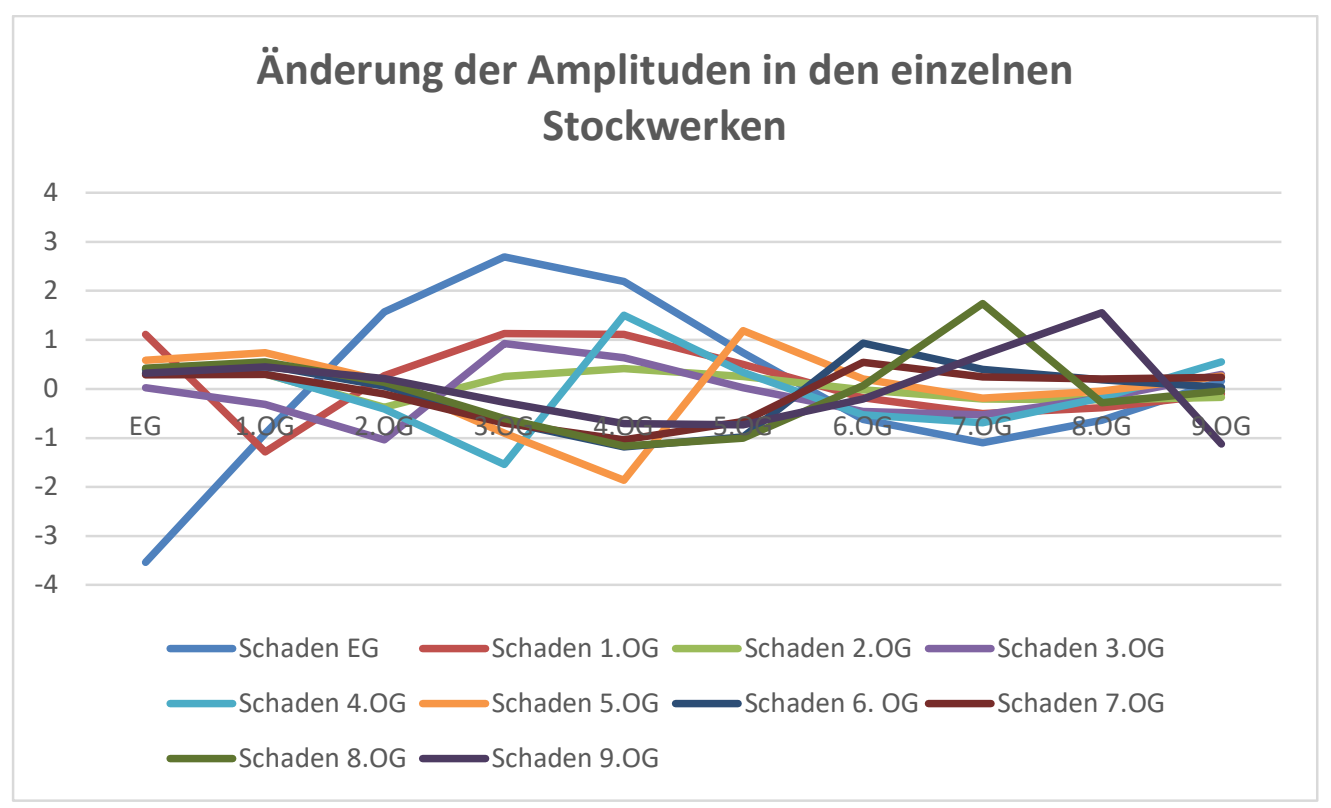

Abbildung 5-5: $\quad 3$. Eigenform: Änderung der Amplituden in den einzelnen Stockwerken

Die Betrachtung von Abbildung 5.5 stimmt zuversichtlich, dass - speziell bei Vorliegen von verlässlichen Informationen über Änderungen der höheren Eigenschwingzustände - eine Interpretation der festgestellten Änderungsmuster hinsichtlich Schadensidentifikation möglich sein sollte.

Unter Ansatz von Einheitslasten in den einzelnen Stockwerkshorizonten wird weiters für den unbeschädigten Zustand die 10 × 10 Nachgiebigkeitsmatrix ermittelt, welche dann mit den derzeit noch in Ausarbeitung befindlichen Ergebnissen aus Abschnitt 5.3 verglichen wird. Da nur Verschiebungen betrachtet werden, liegt eine kondensierte Nachgiebigkeitsmatrix vor, welche sehr wohl die Einflüsse der Knotenverdrehungen im Hauptmodell beinhaltet. Durch Multiplikation mit der Massenmatrix ergibt sich die dynamische Matrix D. Mittels des „MATRIX and Linear Algebra Package for EXCEL" können für jede Variante sofort die verfügbaren 10 Eigenfrequenzen + Eigenformen berechnet werden. Es können nun anschaulich die Änderungen im Vergleich zum unbeschädigten Zustand dargestellt werden.

Es sei ferner darauf verwiesen, dass für jede Variante das $[\Delta \mathrm{K}]$ - die Veränderung der Steifigkeitsmatrix - dargestellt werden kann. Unter der Annahme, dass sich der betrachtete ite Eigenvektor $\left\{\varphi_{i}\right\}$ wenig verändert hat, lässt sich die generalisierte Steifigkeitsänderung

$$
\Delta \mathrm{K}_{\mathrm{i}}^{*}=\left\{\varphi_{i}\right\}^{\top}[\Delta \mathrm{K}]\left\{\varphi_{i}\right\}
$$

\section{Vortrag FLESCH/ KRAMER}

2. Wiener Dynamik Tage 2021 
berechnen, wodurch die zu erwartende Eigenfrequenzänderung abgeschätzt werden kann. Weiters wird mittelfristig die Sensibilität der Änderungen auf unterschiedliche Prozentsätze von lokalen Steifigkeitsänderungen untersucht, um Parameter für ein gezieltes „modelupdating" zu erhalten.

Es ist weiters angedacht, in SOFISTIK ein 3D - Modell des gegenständlichen Gebäudes zu erstellen. Mit diesem Modell kann dann eine gezielte Aufbringung von Schäden an bestimmten Stellen untersucht werden. Für eine künftige praktische Anwendung erscheint es sehr wichtig Erfahrungen zu gewinnen, inwieweit sich lokale Schäden an bestimmten Stellen durch globale Annahmen betreffend die Abminderung von Stockwerkssteifigkeiten darstellen lassen. Bei unsymmetrischen Schäden spielen dann weiters die Torsions-Eigenschwingzustände des Bauwerks eine wichtige Rolle.

\subsection{Analytische Vorgangsweise}

\subsubsection{Allgemeines}

Es wurde ein vereinfachtes analytisches 2D Modell in Excel erstellt. Dabei werden die $\mathrm{x}$ - und y-Koordinaten der Elementknotenpunkte, sowie der E-Modul, die Biege- und Schubsteifigkeit sowie die Querschnittsfläche jedes Elementes eingegeben. Die Eingabeparameter beschränken sich dabei separat auf jedes Element, wodurch die Auswirkung etwaiger Schäden gewisser Elemente auf die Gesamtsteifigkeit des Gebäudes berücksichtigt werden können. Die Vorgehensweise zur Erstellung der globalen Steifigkeitsmatrix erfolgt auf Basis der Finiten Elemente Methode, wobei zuerst die Steifigkeitsmatritzen jedes einzelnen Elementes gebildet werden. Anschließend werden die lokalen Elementsteifigkeitsmatritzen zu einer globalen Systemsteifigkeitsmatrix zusammengeführt. Der Detailierungsgrad des Bauwerkmodells kann somit nach Belieben definiert werden. Je nach Anwendungsbereich sollte die Anzahl der Elemente so gewählt werden, dass die Reduktion der Systemsteifigkeit Aufgrund von Bauwerksschäden ausreichend genau abgebildet werden kann. Das Ziel ist es also ein einfach aufgebautes Excel-Tool zu erstellen, welches überschlagsmäßig die Steifigkeitsmatrix eines Bauwerks erstellt.

\subsubsection{Erstes Beispielmodell mit zwei Elementen}

Als erstes Beispielmodell wurde ein Stabwerksystem bestehend aus einer eingespannten Stütze und einem Deckenbalken gewählt, welche biegesteif miteinander verbunden sind. Es wurde eine horizontale Kraft und ein Moment am Knoten 2 aufgebracht, siehe Abbildung 5-6.

\section{Vortrag FLESCH/ KRAMER}

2. Wiener Dynamik Tage 2021 
In der Excel Datei wurden zunächst die Steifigkeitsmatritzen für jedes der beiden Elemente erstellt, und anschließend zu einer globalen Systemsteifigkeitsmatrix zusammengeführt (siehe Abbildung 5-7).

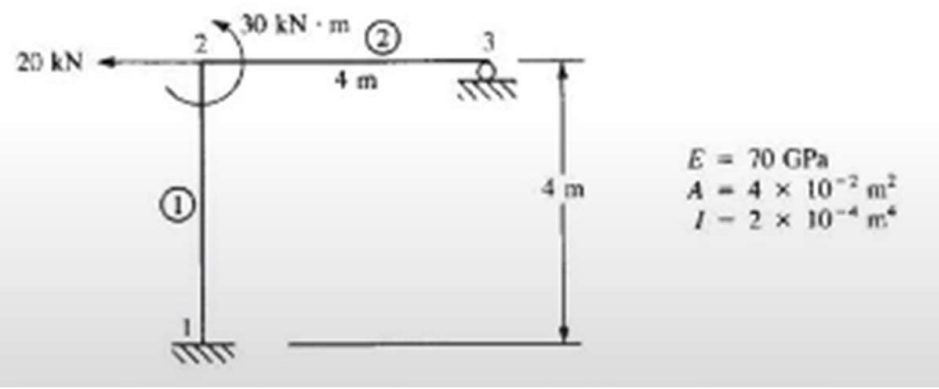

Abbildung 5-6: Systemskizze des ersten Beispielmodelles.

Um die Steifigkeitsmatrix anschließend zu kontrollieren wurde das System nach seinen unbekannten kinetischen und kinematischen Größen gelöst und mit einer Handrechnung auf Basis der Formänderungsenergie verglichen.

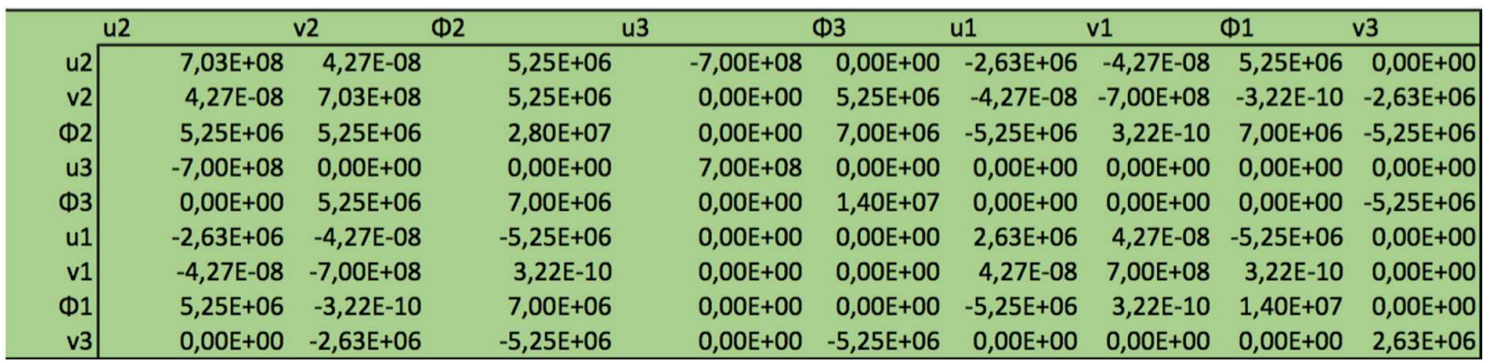

Abbildung 5-7: Steifigkeitsmatrix des ersten Beispielmodelles

Nach dem Löschen der Freiheitsgrade, welche durch die Randbedingungen eingeschränkt werden, konnte die Nachgiebigkeitsmatrix durch das Invertieren der Steifigkeitsmatrix erhalten werden. Anschließend wurden mit dem Excel-Tool sämtliche Eigenfrequenzen und Eigenformen berechnet und können der Abbildung 5-8 entnommen werden.

\section{Vortrag FLESCH/ KRAMER}



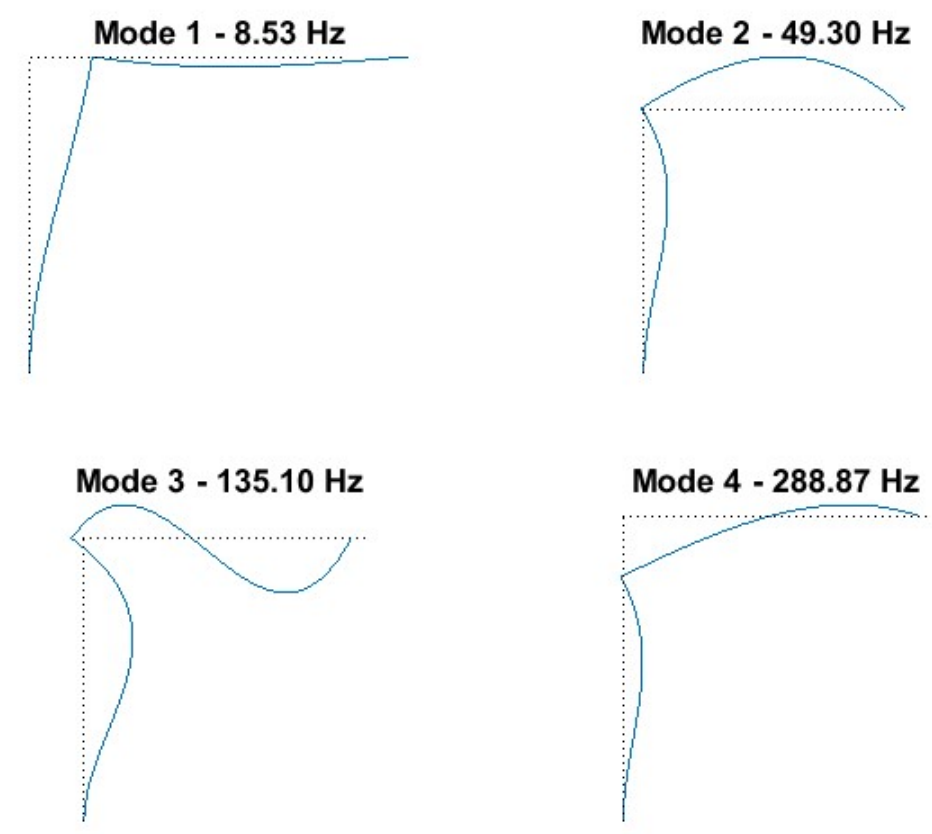

Abbildung 5-8: Die ersten vier Eigenfrequenzen und Eigenformen des Stabwerksystems.

Die Eigenfrequenzen im Schadensfall können also mit Hilfe des Excel Tools berechnet werden. Als Beispiel wurde die Biegesteifigkeit der Stütze um 50\% verringert, wobei der Träger noch die volle Biegetragfähigkeit beibehält. Die Änderung der ersten Eigenfrequenz aufgrund der Reduktion der Biegesteifigkeit der Stütze kann der Abbildung 5-9 entnommen werden.

\begin{tabular}{|c|c|}
\hline \multicolumn{2}{|c|}{ Erste Eigenfrequenz [Hz] } \\
unbeschädigt & beschädigt \\
\hline 8.53 & 6.58 \\
\hline
\end{tabular}

Abbildung 5-9: Änderung der ersten Eigenfrequenz.

\subsubsection{Entwicklung des Demonstrators}

Mit dem Demonstrator können die Auswirkung von lokalen Steifigkeitsminderungen auf die Eigenfrequenzen und Eigenvektoren dargestellt werden. Der Demonstrator dient sowohl zur Veranschaulichung dieser Zusammenhänge im Baudynamik - Unterricht als auch der Entwicklung neuer robuster model - updating - Verfahren.

\section{Vortrag FLESCH/ KRAMER}

\section{Wiener Dynamik Tage 2021}


Die im Abschnitt 5.3.1 beschriebene Vorgangsweise wird nun in der Excel - Datei DEMONSTRATOR in folgenden Varianten aufbereitet:

- Hochbaumodell gemäß Abschnitt 5.1, Untersuchungen analog zu den Untersuchungen mit SOFISTIK

- Brückenmodell.

Zunächst werden nur die Untersuchungen des Hochbaumodells durchgeführt. Bevor die Eigenfrequenzen und Eigenformen berechnet werden, wird eine statische Kondensation der Systemsteifigkeitsmatrix gemäß Abschnitt 15.3 in [1] vorgenommen. Hierdurch erhält man ein dynamisches Ersatzsystem, welches durch die horizontalen Geschoss-VerschiebungsFreiheitsgrade in Y- Richtung beschrieben wird. Durch Invertieren erhält man dann eine Nachgiebigkeitsmatrix, welche auch die Einflüsse der Knotenverdrehungen im Hauptmodell beinhaltet. Durch Multiplikation mit der Massenmatrix ergibt sich die dynamische Matrix $D$. Mittels des „MATRIX and Linear Algebra Package for EXCEL“ können dann 10 Eigenfrequenzen + Eigenformen berechnet werden. Die Berechnungen erfolgen zunächst für das unbeschädigte Gebäude und anschließend für das beschädigte Bauwerk analog zu den Berechnungen in SOFISTIK.

\section{Zusammenfassung}

Die bisherigen Ergebnisse der Berechnungen mit SOFISTIK zeigen anschaulich die unterschiedlichen Änderungsmuster der Eigenfrequenzen und Eigenformen bei Simulation von Schäden in den einzelnen Stockwerken im Bezug auf das unbeschädigte Bauwerk. Am Demonstrator, der analytischen Umsetzung des obigen Konzepts in einem Excel -File, wird derzeit noch gearbeitet. Erste Ergebnisse sind erfolgversprechend. Letztendlich lassen sich die Auswirkungen lokaler Schäden dann im Demonstrator schnell und anschaulich darstellen, ohne parallele Berechnungen z.B. mit dem FE - Programm SOFISTIK vornehmen zu müssen. Der Demonstrator dient sowohl zur Veranschaulichung dieser Zusammenhänge im Baudynamik - Unterricht als auch der Entwicklung neuer robuster model - updating Verfahren.

Zufolge des zunehmenden Trends zum Building Information Modelling (BIM) wird es künftig immer leichter werden, gleichzeitig auch ein Referenzmodell für die dynamische Bauwerksinspektion vorzusehen. Insbesondere für wichtige Infrastruktur sollte man in Zukunft

Vortrag FLESCH/ KRAMER

2. Wiener Dynamik Tage 2021 
für den unbeschädigten Zustand ein Referenzmodell erstellen, welches dann im Katastrophenfall einem raschen und effizienten „model-updating“ zu unterziehen wäre. Um dann für die beschädigte Struktur rasch ein Modell zur Abschätzung der Resttragfähigkeit erstellen zu können, ist die Weiterentwicklung von schnellen und robusten model - updatingStrategien nötig, die sehr wahrscheinlich auch die Verwendung einfacher Ersatzstabmodelle einschließen.

Besonders große und gute Chancen zur Quantifizierung und Lokalisierung von Bauwerksschäden besteht natürlich, wenn im Bauwerk z.B. in jedem Geschoß Sensoren eines Schwingungs-Monitoringsystems installiert sind, die zu jedem Zeitpunkt eine Systemidentifikation erlauben. Die erforderliche Schwingungsanregung wird durch ambiente Schwingungen, Wind und Verkehr geliefert. Will man auch die besonders aussagekräftigen höheren Eigenschwingzustände für die Identifikation heranziehen, könnte man sogar im obersten Stockwerk einen relativ kostengünstigen Schwingungsgenerator installieren, mit dem man zu jedem gewünschten Zeitpunkt eine Anregung mittels Frequenzsweep vornehmen könnte.

\section{Literaturangaben:}

[1] FLESCH R., Baudynamik - praxisgerecht", Band 1, Bauverlag Wiesbaden/ Germany, ISBN 3-7625-3010-6, 1993.

[2] FLESCH R., Baudynamik - praxisgerecht", Band 2, Bauverlag Wiesbaden/ Germany, ISBN 3-7625-3072-6, 1997.

[3] FLESCH R. (Editor): European Manual for In-Situ Assessment of Important Existing Structures. IUSS Press. ISBN: 978-88-6198-006-8, Pavia, Italy. 2007.

[4] FLESCH R., KERNBICHLER K., GRÜBL P.: Brückeninspektion mittels dynamischer Untersuchungen. 8. GESA-Symposium, Duisburg, 1984.

[5] KERNBICHLER K., FLESCH R., RAUSCHER G.: Dynamische Untersuchungen von Großbrücken (Massivbrücken), in-situ-Versuche und Rechenmodelle. Tagung Dynamische Probleme - Modellierung und Wirklichkeit - Univ. Hannover, 1984.

[6] KERNBICHLER K., FLESCH R.: Static and dynamic tests, their qualification for bridge inspection and long-term observations of bridge structures.RILEM- Symposium,Longterm observation of concrete structures, sess.III,Budapest, 1984.

Vortrag FLESCH/ KRAMER

2. Wiener Dynamik Tage 2021 
[7] FLESCH R., KERNBICHLER K., DÜRNSTEINER CH.: Dynamic testing and modelling of Obernberg Bridge. Int. Conf. on num. methods for transient and coupled problems, Venice, 1984.

[8] FLESCH R., KERNBICHLER K., RAUSCHER G.: Dynamische in-situ-Verาsuche und Rechenmodelle - praktische Anwendung auf Großbrücken (Massivbrücken). ÖIAZ, 131. Jg., Heft 10/1986.

[9] FLESCH R., KERNBICHLER K.: Bridge inspection by dynamic tests and calculations dynamic investigations of Lavant Bridge. Int. Workshop on structural safety evaluation based on system identification approaches. Lambrecht/FRG, 1987.

[10] FLESCH R.G., KERNBICHLER K.: A dynamic method for the safety inspection of large prestressed bridges. Int. NSF Workshop "Nondestructive Evaluation for Performance of Civil Struc ᄀtures". Davison Conf. Center. Univ. of South. California, Los Angeles, Februar 1988.

[11] FLESCH R., KERNBICHLER K.: Diagnostic dynamic testing of bridges on Brenner Motorway. Int. conf. on Bridge Management. Guildford/UK. March 1990.

[12] FLESCH R.G., KERNBICHLER K.: A dynamic method for the safety inspection of large prestressed bridges. NATO US-European Workshop on Bridges.

Baltimore/USA. April 1990.

[13] FLESCH R.G.: A dynamic method for the safety inspection of large prestressed bridges. Proc. 2nd Workshop on Bridge. Eng. Research in Progress. Reno/Nevada, Oktober 1990.

[14] FLESCH R.G.:Dynamic testing and system identification for bridge inspection.Proc. of 3rd Int.Workshop on Bridge Rehabilitation , pp. 383 , Ernst \&Sohn ,1992.

[15] FLESCH R. G.: Advanced methods for assessing the seismic vulnerability of existing bridges. Contribution to EU - Japan Workshop, Chania, Crete, March 1998.

[16] FLESCH R.G., STEBERNJAK B., FREYTAG B.: Dynamic insitu testing and FE modelling of bridge WARTH / Austria. ISMA 23, Leuven- Belgium, Sept. 1998.

[17] FLESCH R.G., DUMA G., DARIN E. M., PANZA G.F., ROMANELLI F., CUNHA A., BARBAT A.: Advanced methods for assessing the seismic vulnerability of existing bridges. Contribution to SISM-98 Seismic Impacts on Structures and Monuments, Workshop on Reducing Earthquake Risks to Structures and Monuments in the European Union, Univ. of Cambridge/ UK, November 1998.

[18] FLESCH R.G., STEBERNJAK B., FREYTAG B., MAECK J., OLIA S.: FE-modelling of RC structures within the SIMCES project. 17th IMAC, Kissimmee, Florida, Febr. 1999.

Vortrag FLESCH/ KRAMER

2. Wiener Dynamik Tage 2021 
[19] FLESCH R.G., STEBERNJAK B., FREYTAG B.: System identification of bridge Warth I Austria. EURODYN'99, Prague, June 1999.

[20] FLESCH R.: Die Anwendung der Systemidentifikation zum Monitoring von Bauwerken. D-A-CH Tagung für Erdbebeningenieurwesen, Baudynamik und Windtechnologie, Berlin, Nov. 1999.

[21] FLESCH R.G., et al.: Advanced methods for assessing the seismic vulnerability of existing motorway bridges. 3rd EU - Japan workshop on seismic risk, Kyoto, March 2000.

[22] FLESCH R. G. et al.: „Advanced Methods for Assessing the Seismic Vulnerability of Existing Motorway Bridges. Proc. Workshop Mitigation of Seismic Risk - Support to Recently Affected European Countries. Belgirate, Italy. Nov. 2000.

[23] FLESCH R: In-situ Assessment of Important Buildings and Infrastructure-Background. Proc. 3rd European Conference on Structural Control, July 2004, Vienna.

[24] LU S., RALBOVSKY M., KÖLLNER W., DEIX S., FLESCH R.: Assessment of lifeline Structures in the case of hospitals in seismic zones 3 and 4 in Austria. Proc. 3rd European Conference on Structural Control, July 2004, Vienna.

[25] LU S., RALBOVSKY M., KÖLLNER W., FLESCH R., GRAF H.: Seismic Evaluation of Several Hospitals in Seismic Zones 3 and 4 in Austria. Proc. 13th World Conference on Earthquake Engineering, August 2004, Vancouver.

[26] FLESCH R., LU S., RALBOVSKY M., KÖLLNER W., GRAF H.: Assessment der Erdbebensicherheit von bestehenden Krankenhäusern in Österreichischen Erbebenzonen 3 und 4. Bauingenieur/ D-A-CH Mitteilungsblatt. Ausgabe September 2004/ Band 79. pp. S9-S14.

[27] FLESCH R., et. al.: Dynamic in-situ Measurements, FE - Modelling and Model Updating - Innovative Tools in Civil Engineering. 1st IOMAC, Copenhagen, April 2005.

[28] FLESCH R.; et. al.: The European in-situ Assessment Manual for Important Existing Structures. Proc. 8NCEE, San Francisco, April 2006.

[29] FLESCH R., et al.: In-situ Assessment of Important Existing Structures - IP LESSLOSS/ Sub Project 5 -A Progress Report. Proc. 1stECEES, Geneva, Sept. 2006.

[30] FLESCH R., RALBOVSKY M.: Die Kombination von dynamischen insituUntersuchungen mit FE - Modellierungen. Innovation und Ästhetik _ Festkolloquium zum 75. Geburtstag von Lutz Sparowitz, TU-Graz, 4. Dezember 2015.

Vortrag FLESCH/ KRAMER

2. Wiener Dynamik Tage 2021 
[31] GÖGE, D., LINK, M.: Parametric updating of finite element models by minimizing response residuals at resonances, International Conference on Structural System Identification, Kassel, Germany, p.419-430, 2001.

[32] DOEBLING S.W., FARRAR, C.R., PRIME, M.B.: A Summary Review of VibrationBased Damage Identification Methods, The Shock and Vibration Digest, Vol. 30, No. 2, p. 91-105, 1998.

[33] MAECK, J.: Damage Assessment of Civil Engineering Structures by Vibration Monitoring, PhD thesis, Katholieke Uni-versiteit Leuven, 2003.

[34] ALVIN, K.F.: Finite Element Model Update via Bayesian Estimation and Minimization of Dynamic Residuals, 14th International Modal Analysis Conference, Dearborn, USA, p.561-567, 1996.

[35] PEETERS, B.: System identification and Damage Detection in Civil Engineering, PhD thesis, Katholieke Universiteit Leuven, 2000.

[36] RALBOVSKY, M.: Damage Detection in Concrete Structures Using Modal Force Residual Method. Dissertation Slovak University of Technology in Bratislava, 2007.

[37] RALBOVSKY M., DEIX S., FLESCH R.: "Frequency changes in frequency-based damage identification"; Structure and Infrastructure Engineering, Vol. 6 (2010), Issue 5; S. 611 - 619.

[38] RALBOVSKY M., DEIX S., FLESCH R.: "Probabilistische Betrachtungen der Brückendynamik und Schadenserkennung"; Bauingenieur. D-A-CH Mitteilungsblatt, März 2007 (2007), S. 12 - 16.

[39] FLESCH R., FRIEDL H., KWAPISZ M.: Nachweis der Erdbebensicherheit einer Verbrennungsanlage für radioaktive Abfälle. 12. D-A-CH Tagung Erdbeben und Baudynamik, Hannover, 2011.

[40] NATKE H.G.: Einführung in Theorie und Praxis der Zeitreihen- und Modalanalyse. Vieweg und Sohn, Braunschweig, 1983.

[41] FLESCH R.: Die Methoden der Baudynamik mit spezieller Berücksichtigung ihrer Anwendbarkeit zur Bauwerksinspektion. Habilitationsschrift Teil 1 und 2, TU-Graz, 1988.

[42] DEIX St.: Schadensidentifikation durch experimentelle Modalanalyse und FE-Model Updating. Diplomarbeit Univ. Wien, 2004.

Autorenkontakt: rainer.flesch@ait.ac.at

Vortrag FLESCH/ KRAMER

2. Wiener Dynamik Tage 2021 
Vortrag FLESCH/ KRAMER

2. Wiener Dynamik Tage 2021 